\title{
CoreNEURON: Performance and Energy Efficiency Evaluation on Intel and Arm CPUs
}

\author{
Joel Criado \\ Computer Science dept. \\ Barcelona Supercomputing Center \\ Spain \\ joel.criado@bsc.es \\ Omar Awile \\ Blue Brain Project \\ Ecole Polytechnique Fédérale \\ de Lausanne (EPFL), Switzerland \\ omar.awile@epfl.ch
}

\author{
Marta Garcia-Gasulla \\ Computer Science dept. \\ Barcelona Supercomputing Center \\ Spain \\ marta.garcia@bsc.es \\ Ioannis Magkanaris \\ Blue Brain Project \\ Ecole Polytechnique Fédérale \\ de Lausanne (EPFL), Switzerland \\ ioannis.magkanaris@epfl.ch
}

\author{
Pramod Kumbhar \\ Blue Brain Project \\ Ecole Polytechnique Fédérale \\ de Lausanne (EPFL), Switzerland \\ pramod.kumbhar@epfl.ch \\ Filippo Mantovani \\ Computer Science dept. \\ Barcelona Supercomputing Center \\ Spain \\ filippo.mantovani@bsc.es
}

\begin{abstract}
The simulation of detailed neuronal circuits is based on computationally expensive software simulations and requires access to a large computing cluster. The appearance of new Instruction Set Architectures (ISAs) in most recent HighPerformance Computing (HPC) systems, together with the layers of system software and complex scientific a pplications running on top of them, makes the performance and power figures challenging to evaluate. In this paper, we focus on evaluating CoreNEURON on two HPC systems powered by Intel and Arm architectures. CoreNEURON is a computational engine of the widely used NEURON simulator adapted to run on emerging architectures while maintaining compatibility with existing NEURON models developed by the neuroscience community. The evaluation is based on the analysis of the dynamic instruction mix on two versions of CoreNEURON. It focuses on the performance gain obtained by exploiting the Single Instruction Multiple Data (SIMD) unit and includes energy measurements. Our results show that using a tool for increasing data-level parallelism (ISPC) boosts the performance up to $2 \times$ independently on the ISA. Its combination with vendor-specific compilers can further speed up the neural simulation time. Also, the performance/price ratio is higher for Arm-based systems than for Intel ones making them more cost-efficient keeping the same u sability level of other HPC systems.
\end{abstract}

Index Terms-NEURON, neuronal networks, supercomputing, performance evaluation, energy efficiency, A rm, I ntel, SIMD

\section{INTRODUCTION AND RELATED WORK}

Even though research in neuroscience is advancing at a rapid pace, acquiring a fundamental understanding of the brain remains an open challenge. The neuroscience community is developing various tools and techniques to understand the cellular mechanisms of brain functions. As part of these efforts, the computational modelling community focuses on developing mathematical models of neuron cells based on experimental data. With the availability of more experimental data, neuroscientists are building more detailed neuron models of brain tissue. For example, as part of the European Human Brain Project (HBP), researchers are developing a full-scale model of a rat hippocampus CA $1^{1}$. This model has about 447 thousand neurons, 304 million compartments, and 990 million synapses. To study such models at different scales, the community has developed various simulation software such as NEURON [1] for morphologically detailed neuron models, NEST [2] for point neuron models, and STEPS [3] for simulations at the molecular level.

The simulation of morphologically detailed neuronal circuits like rat hippocampus CA1 is computationally expensive and requires access to a large computing cluster. Analyzing and optimizing such simulation software's performance on different hardware platforms is essential for delivering scientific results faster, and reducing the computational cost of such large scale simulations. In this paper, we present our efforts to analyze the performance of CoreNEURON [4], a compute engine of widely used NEURON simulator. Specifically, by using newly developed NMODL source to source compiler framework [5] with ISPC backend [6], we analyzed different performance metrics to evaluate Intel and Arm platforms. For decades scientific computing has been associated with mostly a single architecture, Intel x86. Since November 2018, the Armv8 architecture is part of the Top500 list ${ }^{2}$ with the Astra supercomputer [7]. In June 2020, Fugaku by Fujitsu powered by the Arm Instruction Set Architecture (ISA), has been ranked as the fastest supercomputer in the world. Therefore, a valid question is how well complex applications such as neural simulations behave on high-performance systems powered by different architectures? There are several approaches in the literature [8], [9], mostly targeting Arm mobile SoCs. We employ server-grade Arm CPUs similar to [10], [11], but evaluating a different workflow.

We isolated three layers that can affect the performance

\footnotetext{
${ }^{1}$ Human Brain project, Community Models of Hippocampus https://www.humanbrainproject.eu/en/brain-simulation/hippocampus/

${ }^{2}$ https://www.top500.org/lists/top500/2018/11/
} 
and the energy efficiency of scientific applications running on large computing systems. First, the hardware, which includes all the components and their integrations. We tackle this layer studying two systems powered by two different ISAs: $\mathrm{x} 86$ by Intel and Armv8 by Arm. Second, the system software, which includes the OS, the compilers, and all the libraries required by the application to run. We focused on compilers within this layer, comparing GCC with vendor-specific compilers (by Intel and Arm). Third, the scientific application, as seen from the computer scientist. Therefore, this includes the approach used by the developers for leveraging the layers mentioned above. We evaluate a neuroscience simulator that takes advantage of the ISPC compiler, a tool developed for leveraging data-level parallelism on different technologies, including Intel and Arm. Sprenger et al. in [12] evaluates the same tool but for speeding up database operations.

We complement our evaluation studying how these layers affect two fundamental aspects of modern high-performance computing systems: $i$ ) the performance gain given by vectorization and $i$ ) the energy consumption of neural simulation.

Vectorization, in our case, careful use of the SIMD units, is under scrutiny by the whole HPC community [13] due to its increasing popularity pushed by the introduction of the Scalable Vector Extension (SVE) of Arm, the SX-Aurora vector accelerator by NEC [14] and the V-extension of the RISC-V ISA. Given the importance of this topic, we investigate the dynamic instruction mix of our application to quantify how this form of data-level parallelism is leveraged by the application and the compiler on different ISAs. Energy consumption is another hot topic. Therefore we complement our study with energy measurement changing parameters on all three layers mentioned above, showing how important the system software layer is not only for performance but also for energy consumption.

The main contributions of this paper are: $i$ ) we present an indepth study of the dynamic instruction mix of two versions of the CoreNEURON code on both ISAs; $i$ ) we provide figures of performance and energy consumption measured on stateof-the-art HPC computing infrastructures; iii) we focused on performance gain obtained by efficient use of SIMD units, exposing potential gain for the new vector extensions such as the Arm Scalable Vector Extension (SVE) [15].

The rest of the document is organized as follows. Section II summarizes the environment employed for the evaluation, detailing the software infrastructure, and the hardware of the computational platforms. Section III introduces the methodology followed for the evaluation. In Section IV, we report the measurements gathered from our experiments, including an analysis of the instruction mix correlated with the performance of the codes under study on different clusters. This section also includes energy measurements and a comment about the costefficiency of the evaluated platforms. Section V concludes our study with comments and remarks.

\section{ENVIRONMENT}

\section{A. NEURON, CoreNEURON and NMODL}

NEURON is a simulation environment developed over the last three decades for modeling networks of neurons with complex branched anatomy and biophysical membrane properties. It enables simulations of detailed neuron models on computing platforms from desktop to large supercomputing platforms. To improve the performance and adapt the codebase for modern computing architectures, the main simulation algorithms of NEURON were refactored into a new simulation engine called CoreNEURON. One of the critical features of the NEURON simulator is extendability via a domain-specific language called NMODL [16]. Users describe neuron mechanisms using NMODL, which gets translated into $\mathrm{C}++$ code using a sourceto-source compiler called MOD2C ${ }^{3}$.

One of the significant limitations of MOD2C is that it lacks the flexibility to generate code for different parallel programming models and several domain-specific optimizations. Also, it relies heavily on vendor compilers (e.g., Intel, Cray, PGI) for auto-vectorization optimization. As the generated code often accounts for more than $80 \%$ of overall execution time, this heavily limits the performance of simulations. To address these limitations, the NMODL source-to-source compiler framework has been developed. NMODL takes benefit of modern compiler techniques to generate efficient code. It takes MOD files written in NMODL as an input and converts it into intermediate Abstract Syntax Tree (AST) representation. After various domain-specific transformations and optimizations at the AST level, the code generator emits code into different backends like C++, OpenMP, OpenACC, or ISPC. The overall architecture of the NEURON simulator with CoreNEURON and NMODL is shown in Figure 1.

Until recently, CoreNEURON was relying on compiler autovectorization to take advantage of SIMD extensions of target hardware platforms. This has proven [4] to work particularly well on x86 platforms and using the Intel Parallel Studio $\mathrm{C} / \mathrm{C}++$ compilers. Unfortunately, auto-vectorization performance using other compilers (e.g., GCC, clang) has been suboptimal or impossible for the CoreNEURON kernels, even on $x 86$ hardware platforms. This was previously tested on Intel Skylake and Intel KNL [17].

In this study, we used ISPC backend in NMODL. The Intel SPMD Program Compiler (ISPC) is an open-source, compiler framework for SIMD programming on the CPU. It is built on top of the LLVM compiler framework, supporting major vector extensions on x86 (SSE4.2, AVX2, AVX-512), ARM (NEON), and Nvidia GPUs (NVPTX). For this study, we used a synthetic model consisting of a multiple ring network of branching neurons [18]. This model is developed to help in performance characterization with an easy parameterization for the number of cells, branching pattern, compartment per branch, etc.

\footnotetext{
${ }^{3}$ Blue Brain Project, MOD2C - Converter for Mod Files to C Code, http://github.com/BlueBrain/mod2c
} 


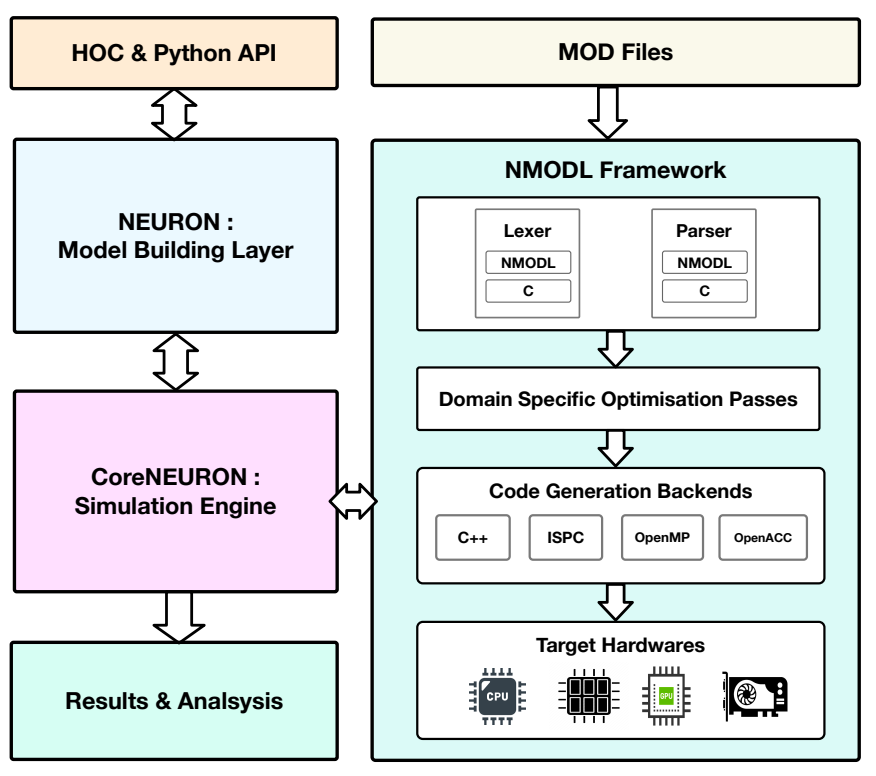

Fig. 1. An overview of the NEURON-CoreNEURON simulator toolchain (on the left) and NMODL source-to-source compiler framework used for DSL to $\mathrm{C}++$ code generation targeting various hardware platforms (on the right).

\section{B. Hardware environment}

The two clusters employed for the evaluation of this paper are MareNostrum4 and Dibona. MareNostrum4 is a Tier-0 supercomputer in production at the Barcelona Supercomputing Center (BSC) in Barcelona, Spain. It is powered by Intel x86 Skylake CPUs, each with 24 cores. Dibona is an Armbased experimental cluster based on the Bull/ATOS Sequana infrastructure with compute nodes powered by two Marvell ThunderX2 CN9980 processors, each with 32 Armv8 cores. More details about the Dibona cluster can be found in [19], [20]. Table I shows the details of the hardware configurations of the two platforms. Both CPU technologies offer Turbo Boost and simultaneous multithreading (SMT) configurations even if we do not use these options for our tests.

TABLE I

HARDWARE CONFIGURATION OF THE HPC PLATFORMS

\begin{tabular}{l|c|c} 
& Dibona-TX2 & MareNostrum4 \\
\hline Core architecture & Armv8 & Intel x86 \\
CPU name & ThunderX2 & Skylake Platinum \\
CPU model & CN9980 & 8160 \\
Frequency [GHz] & 2.0 & 2.1 \\
Sockets/node & 2 & 2 \\
Core/node & 64 & 48 \\
SIMD vector width & 128 & $128 / 256 / 512$ \\
Mem/node [GB] & 256 & 96 \\
Mem tech & DDR4-2666 & DDR4-3200 \\
Mem channels/socket & 8 & 6 \\
Num. of nodes & 40 & 3456 \\
Interconnection & Infiniband EDR & Intel OmniPath \\
System integrator & ATOS/Bull & Lenovo
\end{tabular}

In Dibona, the power monitoring infrastructure allows us to homogeneously measure the power consumption of the whole computational node (including the CPU, the memory, the network adapters, the I/O devices, and most of the motherboard). The Sequana infrastructure underneath Dibona allows to house compute nodes powered by both, Arm and Intel CPUs. The compute node infrastructure is engineered to be similar for both architecture, and most importantly, the power monitoring infrastructure is the same when switching CPU type. The Intel nodes used for the energy measurements reported in Section IV-C are Skylake Platinum 8176 (with 28 core per socket). They have been plugged in the Sequana infrastructure exclusively for gathering the energy measurements in a fair, trusted and homogeneous way.

\section{Methodology}

As stated in Section I, several layers of the HPC stack affect the performance of a given software in an HPC environment. In our current scenario, testing a neuron model simulation using HPC clusters with different ISAs, we consider that the main factors affecting the performance are:

- Hardware: x86 vs. Armv8.

- Compiler: Generic (GCC) vs. vendor-specific (Intel, Arm HPC compiler).

- Application: ISPC vs. no ISPC.

These are the three variables that we use in all analyses performed in this article.

All executions use the same version of CoreNEURON (0.17 commit [42da29d]), NMODL (0.2 commit [9202b1e]) and ISPC (1.12.0). We employed the ringtest model [18] as the input for every run. The system software used has been selected to be as similar as possible on both systems. In Table II, we can see the details of the used versions. For compilers, no special flags have been added besides the ones already provided by each software component and -03 .

TABLE II

CLUSTERS SOFTWARE ENVIRONMENT

\begin{tabular}{l|c|c} 
& Dibona-TX2 & MareNostrum4 \\
\hline GCC & GCC 8.2.0 & GCC 8.1.0 \\
Vendor compiler & arm 20.1 & icc 2019.5 \\
MPI lib. & OpenMPI 3.1.2 & IMPI 2017.4 \\
PAPI & PAPI 5.6.1 & PAPI 5.7.0 \\
Tracing & Extrae 3.5.4 & Extrae 3.7.1 \\
CoreNEURON & $0.17[42 \mathrm{da} 29 \mathrm{~d}]$ & $0.17[42 \mathrm{da} 29 \mathrm{~d}]$ \\
NMODL & $0.2[9202 \mathrm{~b} 1 \mathrm{e}]$ & $0.2[9202 \mathrm{~b} 1 \mathrm{e}]$ \\
ISPC & 1.12 & 1.12
\end{tabular}

All executions use a full node, 64 cores in Dibona, and 48 in Marenostrum4, using the MPI only parallelization with processes pinned contiguously.

As seen in a previous work [5], CoreNEURON simulation time is dominated by the current and state update kernels of the various mechanisms (can be found in the code as nrn_cur_hh and nrn_state_hh). These kernels are computationally intensive and account for more than $90 \%$ of the total instructions executed for the ringtest model. We gather all measurements that we present from these two kernels.

The evaluation is divided into different steps and starting from a top-bottom approach. We start from a general 
performance overview of global metrics such as execution time, the number of instructions executed, the number of cycles consumed, and average IPC. For the execution time, we performed five isolated runs for each set of parameters and reported the average value of the elapsed time. In all cases, the relative error is below 5\%. Therefore, we do not show error bars on the charts for the sake of clarity.

Since the main improvements expected from the use of the ISPC are related to leveraging SIMD instructions, we include an analysis of the dynamic instructions mix to obtain more insight into the global metrics.

TABLE III

HardWARE COUnTERs on MARENostrum4 (MN4) AND Dibona (DB)

\begin{tabular}{|c|c|c|}
\hline MN4 & DB & PAPI Hardware counter \\
\hline$\checkmark$ & $\checkmark$ & PAPI_TOT_INS: Total instr. executed \\
\hline$\checkmark$ & $\checkmark$ & PAPI_TOT_CYC: Total cycles used \\
\hline$\checkmark$ & $\checkmark$ & PAPI_LD_INS: Total load instr. executed \\
\hline$\checkmark$ & $\checkmark$ & PAPI_SR_INS: Total store instr. executed \\
\hline \multirow[t]{3}{*}{$\checkmark$} & $\checkmark$ & PAPI_BR_INS: Total branch instr. executed \\
\hline & $\checkmark$ & PAPI_FP_INS: Total floating point instr. executed \\
\hline & $\checkmark$ & PAPI_VEC_INS: Total vector instr. executed \\
\hline$\checkmark$ & & PAPI_VEC_DP: Total vector instr. double precision exec. \\
\hline
\end{tabular}

To obtain the instruction mix, we use Extrae [21] and PAPI [22], we have adequately instrumented the application with Extrae to gather metrics just from the two relevant kernels (nrn_cur_hh and nrn_state_hh). The PAPI counters available in both systems are slightly different. In Table III, we list the exact hardware PAPI counters gathered in each system and their description.

Finally, since energy consumption is a hot topic in the HPC domain nowadays, we incorporate an energy comparison of both architectures. To obtain the energy measurements, we take advantage of Dibona's unique infrastructure, which includes the same cluster nodes with different ISAs (x86 and Armv8) sharing the same power monitoring infrastructure. This configuration makes the energy measurements fair and comparable. The energy measurements are obtained taking into account the main computation phase disregarding the initialization and setup of the simulation Again, five independent runs were performed, and their average is used, the relative error is below $5 \%$, so we do not show error bars for simplicity.

\section{EVALUATION}

\section{A. Performance comparison}

In this subsection, we present the global performance metrics gathered for the different runs. In Figure 2, we can see the elapsed time of a CoreNEURON simulation and the average IPC of the execution with the different configurations. In the case of $\mathrm{x} 86$, the ISPC version compiled with GCC and Intel takes the same time as the No ISPC version compiled with Intel. This confirms that the Intel compiler can obtain the same performance with and without ISPC. On the other side, we see that the ISPC version with the GCC compiler achieves the same execution time as the Intel compiler. In comparison, the No ISPC version compiled with GCC is more than two times slower than the same version compiled with Intel.

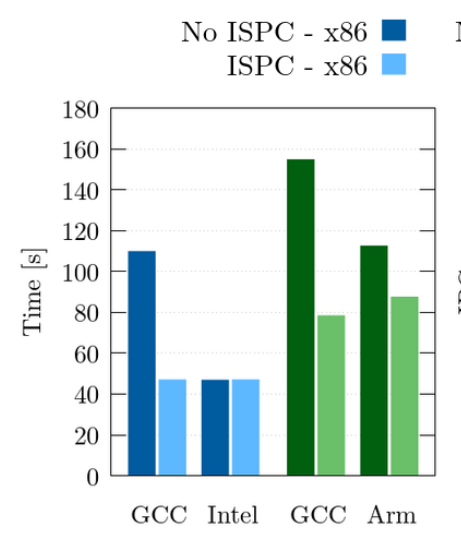

No ISPC - Arm

ISPC - Arm

Fig. 2. Execution time using GCC and Vendor compilers

In the case of Armv8, the use of ISPC improves the execution of the No ISPC for both compilers. When using ISPC GCC and Arm HPC compiler, the execution time is similar but slightly better for the version compiled with GCC. If we look at the execution time of the No ISPC in Armv8, we can say that the Arm HPC compiler can generate a more efficient code than GCC. When using the GCC compiler, the ISPC version reduces the execution time to half.

Looking at the plot on the right-hand side of Figure 2, we can see the average IPC of each execution. The most eyecatching fact is that the executions in $\mathrm{x} 86$ when using the Intel compiler have a very different IPC when using the ISPC and No ISPC, while they presented almost the same execution time. In the case of Armv8, the IPC obtained by the ISPC version is the same for both compilers. The IPC of the No ISPC version is better than the ISPC version. All seems to indicate that the ISPC version can generate code that exploits the SIMD units better, as in all the cases, ISPC is faster but with a lower IPC. In Section IV-B we present more detailed metrics to understand where the difference comes from.

In Figure 3, we can see the number of instructions executed during one simulation and the number of cycles consumed by the different versions.

If we compare the right-hand side of the plot containing the number of cycles with the left-hand side of Figure 2 showing the elapsed time, we can observe that they have the same trend. This tells us that the elapsed time is directly proportional to the number of cycles consumed, meaning that the frequency is constant as expected.

However, this is not true for the number of instructions, the left-hand side of Figure 3. The number of instructions executed by the ISPC version seems to be independent of the compiler, the ISPC version compiled with GCC and executed in $\mathrm{x} 86$ executes the same number of instructions as the ISPC version compiled with Intel in the same architecture. The same happens when running in Armv8; the ISPC version executes the same number of instructions when compiled with Arm HPC compiler and GCC. No ISPC version is the opposite; in both architectures, the vendor compiler (i.e., Arm HPC 


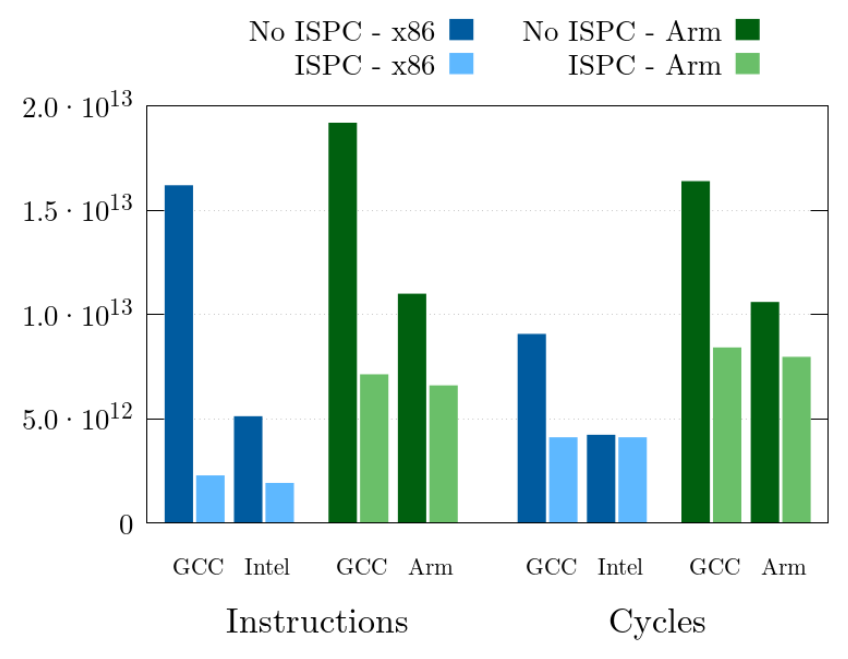

Fig. 3. Number of instructions and cycles used

compiler and Intel) executes fewer instructions than the same version compiled with GCC.

In the case of $\mathrm{x} 86$, the ISPC version executes $14 \%$ of the instructions executed by the No ISPC version when using GCC to compile. In the case of Armv8 the ISPC version executes $37 \%$ of the instructions executed by the No ISPC version when using the GCC compiler.

We can say that the number of total instructions executed by the ISPC version is reduced drastically with respect to the number of instructions executed by the No ISPC version. We suspect that this reduction is due to the use of vector units that can operate with longer vectors and more optimized code. We verify this assumption with the instruction mix evaluation in the following Section.

TABLE IV

PERFORMANCE METRICS FOR RUNS IN BOTH ARCHITECTURES, USING DIFFERENT COMPILERS AND CODE VERSIONS

\begin{tabular}{lllrrrr} 
Arch. & Comp. & Version & Time[s] & Instr. & Cycles & IPC \\
\hline \multirow{4}{*}{ x86 } & \multirow{2}{*}{ GCC } & No ISPC & 109.94 & $16.24 \mathrm{E}+12$ & $9.07 \mathrm{E}+12$ & 1.79 \\
& & ISPC & 47.10 & $2.28 \mathrm{E}+12$ & $4.11 \mathrm{E}+12$ & 0.56 \\
& \multirow{2}{*}{ Intel } & No ISPC & 46.95 & $5.12 \mathrm{E}+12$ & $4.22 \mathrm{E}+12$ & 1.21 \\
& & ISPC & 47.13 & $1.92 \mathrm{E}+12$ & $4.10 \mathrm{E}+12$ & 0.47 \\
\hline \multirow{3}{*}{ Arm } & \multirow{2}{*}{ GCC } & No ISPC & 154.89 & $19.15 \mathrm{E}+12$ & $16.41 \mathrm{E}+12$ & 1.17 \\
& & ISPC & 78.52 & $7.13 \mathrm{E}+12$ & $8.42 \mathrm{E}+12$ & 0.85 \\
& \multirow{2}{*}{ Arm } & No ISPC & 112.64 & $11.05 \mathrm{E}+12$ & $10.57 \mathrm{E}+12$ & 1.04 \\
& & ISPC & 87.64 & $6.59 \mathrm{E}+12$ & $7.96 \mathrm{E}+12$ & 0.82
\end{tabular}

For completeness, we show in Table IV the metrics plotted in Figures 2 and 3.

We also observe that the use of SIMD units handling wider vectors and the more optimized code comes with the price of a reduction in the IPC by more than $2 / 3$. This explains why the reduction in the number of instructions is not reflected proportionally in the reduction of execution time.

\section{B. Instruction mix comparison}

In this section, we analyze the dynamic instruction mix of the different executions using the PAPI counters shown in Table III. We find two kinds of plots in this section, the absolute number of instructions executed and the percentage of each type of instruction.

We can see the instruction mix for both versions, ISPC and No ISPC, when running in Dibona using GCC (top) and Arm (bottom) as a percentage of the total number of instructions in Figure 4. The main difference between the ISPC version and the No ISPC version for both compilers is the presence of vector instructions. While the No ISPC code does not generate vector instruction (less than $0.1 \%$ ), with the ISPC version, more than $50 \%$ of the executed instructions are vector instructions.

Also, the floating-point instructions (FP Ins) account for more than $30 \%$ of the total instructions executed with the No ISPC version, while the ISPC code issues less than $9 \%$ of FP instructions. This data indicates that most of the floating-point instructions performed by the No ISPC version are vectorized in the ISPC version.

If we compare the No ISPC version with GCC and the No ISPC version with Arm HPC compiler, we see that the Arm HPC compiler issues almost two times less instructions. Moreover, this reduction is not due to the use of vector instructions. It is quite a proportional reduction in all types of instructions. As shown in the previous section, both runs still present a similar IPC. We can see that the ISPC version with GCC has the same distribution of instructions as the ISPC version compiled with the Arm HCP compiler.
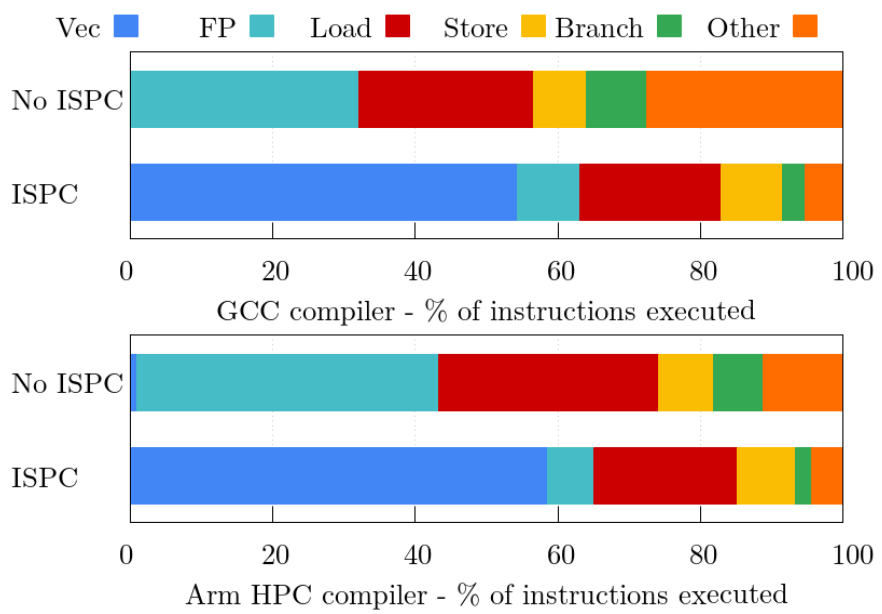

Fig. 4. Percentage of instructions using GCC and Arm HPC compiler on Armv8

In Figure 5, we can see the same data plotted as absolute numbers of instructions executed for the selected kernels and each version of the code.

As expected, we can observe that the total number of instructions has decreased; the ISPC code executes almost three times less instructions than the No ISPC version when using the GCC compiler and two times less when using Arm HPC compiler.

We see that ISPC enables a reduction of executed instructions (all kinds of them). The NEON SIMD unit implemented 

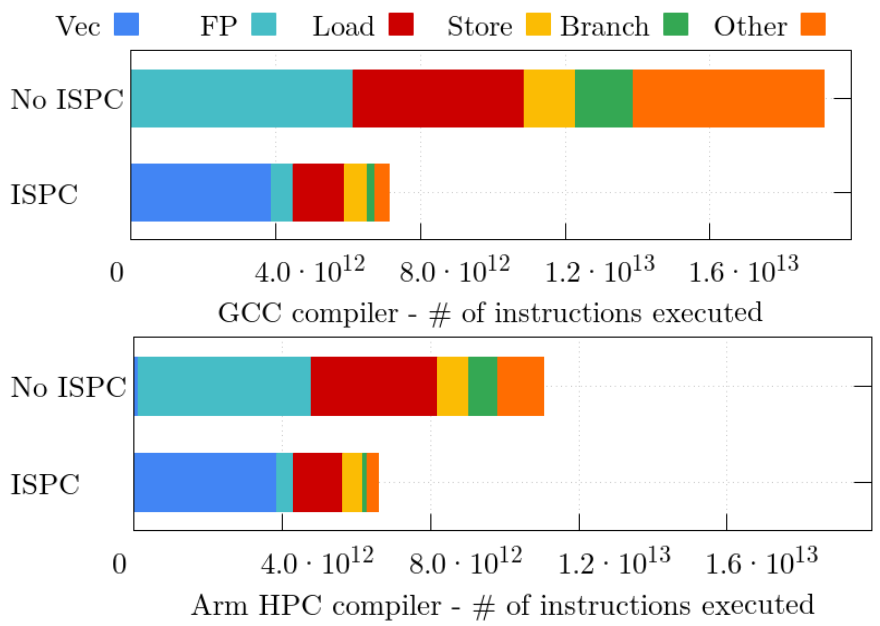

Fig. 5. Instruction mix using GCC and Arm HPC compiler on Armv8

in the ThunderX CPU offers vectors of 128 bits. Since the program uses double-precision numbers, these SIMD units can process up to two double-precision floating-point operations per instruction. An operation can be an algebraic operation, load, or store from memory. As a consequence, one could expect up to half floating-point, load, and store instructions due to the SIMD unit's use. We report therefore the ratio $r_{t}=\frac{i_{t}}{n i_{t}}$, where $t$ is the type of instructions ( $t=s a$ scalar arithmetic, $t=v a$ vector arithmetic, $t=l$ load, $t=s$ store), $i_{t}$ is the number of instructions of type $t$ executed enabling ISPC and $n i_{t}$ is the number of instructions of type $t$ when running the No ISPC code. We observe that $r_{s a+v a}=0.73, r_{l}=0.30$, and $r_{s}=0.43$. This indicates a significant improvement from the use of the vector units and that the code generated by the ISPC version is optimized in the number of branches that it executes and the number of instructions issued.

In Figure 6, we can find the instruction mix for both ISPC and No ISPC as a percentage of instructions respect the total number of instructions executed by the kernel, using GCC (top) and Intel (bottom) when running in the x86 cluster.
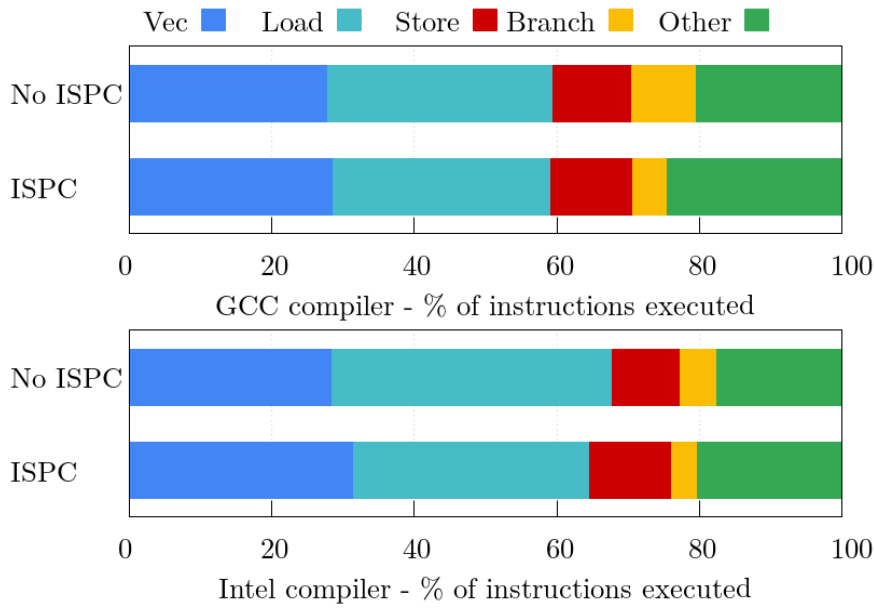

Fig. 6. Percentage of instructions using GCC and Intel compiler on x86
We observe that the ratio of instructions of each type is very similar for both versions, $\sim 27 \%$ of floating-point vector instructions, $\sim 30 \%$ of load instructions, and $\sim 11 \%$ of store instructions.

We can see an essential difference with the results obtained in Armv8, where the No ISPC version was not able to generate any vector instruction; in $\mathrm{x} 86$, the No ISPC version issues $27 \%$ of vector instructions.

Figure 7 shows the total number of instructions of each type executed when running in x86 using GCC (top) and Intel (bottom) for both versions of the code in absolute numbers.
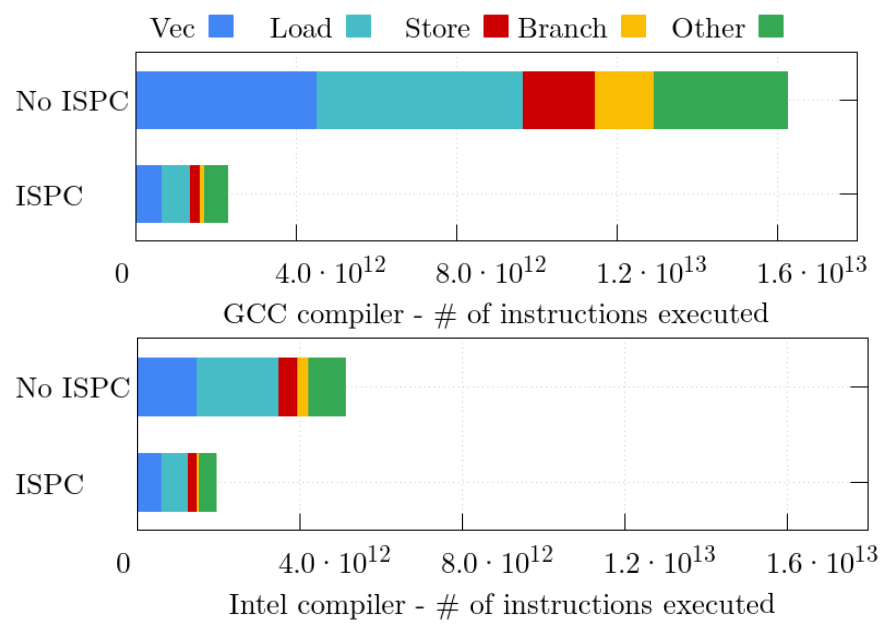

Fig. 7. Instruction mix using GCC and Intel compiler on $\mathrm{x} 86$

Using the GCC compiler, we observe a significant reduction in the number of instructions executed by the ISPC with respect to the number of instructions executed by the No ISPC version, being seven times less instructions. We notice that the reduction does not come from a single type of instruction; all types of instructions are reduced in a similar ratio.

To understand where the reduction in the number of instructions comes from, we manually analyze the binaries of both codes. We study the kind of instructions that each of them contains. Although a study of the dynamic instruction mix is more accurate in the number of instructions executed than studying the static instruction mix, in this case, an analysis of the static instruction mix gives us more insight into the kind of instructions that appear.

Studying the static binaries, we observe that the No ISPC binary contains mostly instructions that use the SSE SIMDextension (128-bit SIMD registers able to operate on two doubles with a single instruction). In contrast, the ISPC binary includes mostly instructions using the AVX-512 SIMDextension (512-bit SIMD registers). The SSE unit can operate on two double-precision operands, and the AVX-512 unit of the $x 86$ processor being used can operate on eight doubleprecision operands on each instruction.

The static analysis explains most of the reduction in the number of instructions of floating-point vector operations, loads, and stores from memory. However, looking at the 
drastic reduction in the number of branches (the ISPC version executes only $7 \%$ of branches executed in the No ISPC version), we can conclude that the ISPC version generates a more optimized code in terms of the number of branches and number of instructions executed. A similar explanation applies to the lower part of Figure 7 corresponding to the Intel compiler's use. The static analysis of the binary of the No ISPC version of the code compiled with the Intel compiler shows in fact that it uses several AVX2 instructions.

Since AVX2 SIMD extension leverages registers of 256 bits, a more advanced extension with registers twice wider than $\mathrm{SSE}$, we expect that this justifies the drastic reduction of this version of the code when compared with the GCC version.

We observed that when enabling ISPC, both compilers take advantage of the AVX512 extension. AVX512 extension leverages registers twice the size of AVX2 extension; this explains almost all the difference in the number of instructions between ISPC and No ISPC when using the Intel compiler.

We also noticed that the use of the Intel compiler generates more complex static binaries that translate into less instructions executed.

\section{Energy Efficiency Comparison}

In this Section, we report the energy consumption of a CoreNEURON execution.

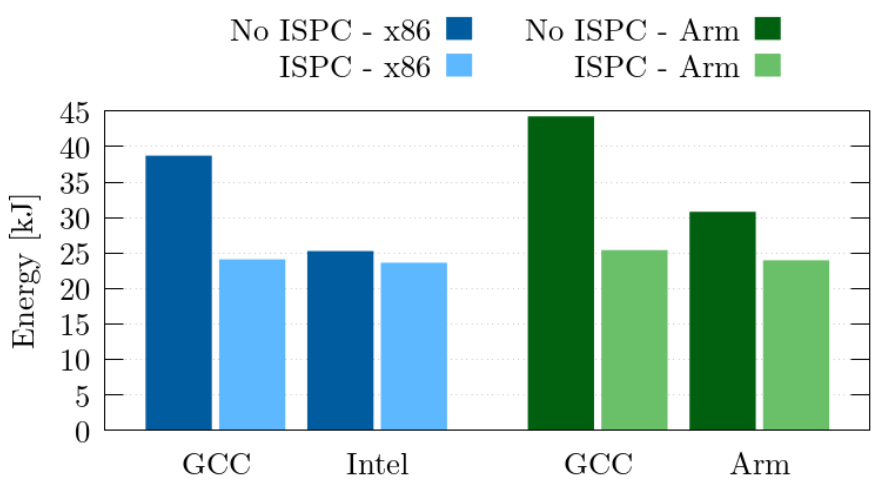

Fig. 8. Energy using GCC and Vendor compilers

Figure 8 shows the energy spent in one simulation using a single node, respectively, powered by x86 Intel Skylake and Armv8 Marvell ThunderX2 CPUs. We see a strong correlation between the energy measurements and the execution time depicted in Figure 2. The trivial observation is that the most efficient architecture is the fastest one. As a consequence, we note that vendor-specific compilers allow to reach a lower energy-to-solution.

More interestingly, we point out that the ISPC version of CoreNEURON requires the same amount of energy on all architectures, even if on Armv8 the execution time is longer than $\mathrm{x} 86$. For this reason, we analyze in Figure 9 the aggregated power drain of the same executions computed as the ratio between the energy and the execution time. We can notice that the average power drain of an $\mathrm{x} 86$ node is $\sim 433 \pm 30 \mathrm{~W}$ while on an Armv8 node is $\sim 297 \pm 14 \mathrm{~W}$. It

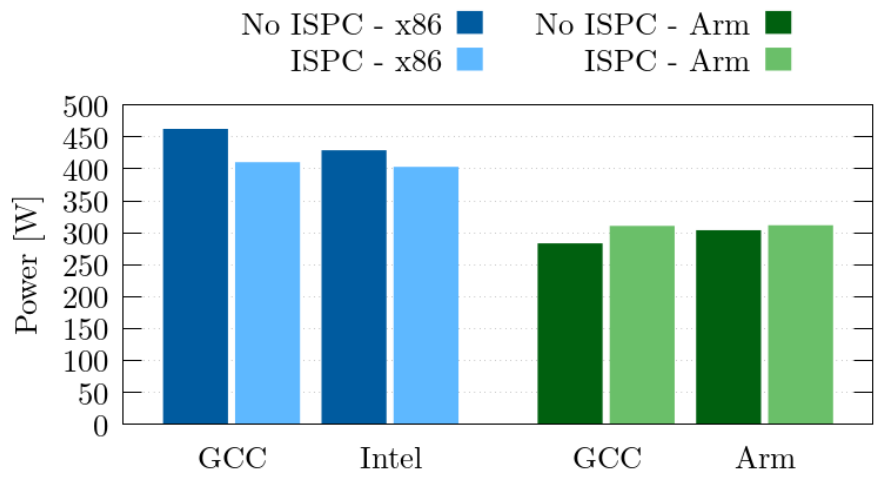

Fig. 9. Power using GCC and Vendor compilers

is interesting to observe that on Armv8 the slowest execution (No ISPC w GCC) correspond to the lowest power drain. This is not true on $\mathrm{x} 86$ nodes. Since we observed in Section IV-B that No ISPC version of the code compiled with GCC does not take advantage of the NEON SIMD extension, we can guess that the Marvell CPU's internal power manager can save some power when the vector unit is not used.

\section{Cost/performance comparison}

Besides the technical analysis that brings us to conclude that the overall performance of the Arm-based system powered by Marvell ThunderX2 CPUs is systematically lower than the Intel Skylake one, we can analyze the cost efficiency of those two systems.

In May 2018, Marvell announced the general availability of the ThunderX2 CPU, with a recommended maximum retail price of $\$ 1795$ each $\mathrm{CPU}^{4}$ (for the configuration we have installed on Dibona with 32 cores). On the Intel website ${ }^{5}$ we retrieve the recommended retail price of the Skylake CPU powering Marenostrum: \$4702. A similar cost-efficiency analysis of Arm and Intel systems is provided by Simon McIntosh Smith et al. in [23]. We decided to focus on the objective price of the CPUs setting explicitly aside the integration expenses because they are often a result of negotiation and, therefore, less objective.

We define the cost efficiency $e=p / c$, where $c$ is the cost of the CPU as presented above and $p$ is the performance of the application under study. We express the performance $p=1 / t$ as the inverse of the time spent simulate as presented in Figure 2. For simplicity, we scale the cost efficiency $e$ by a factor $10^{6}$. We report in Figure 10 the detailed costefficiency based on recommended retail prices. Even assuming some uncertainty on the price, the cost efficiency of a system powered by Armv8 Marvell ThunderX2 CPU is consistently higher (up to $85 \%$ more) than the x 86 Intel Skylake one. If we focus on the fastest versions of the codes (i.e., ISPC using vendor-specific compilers), we notice that the cost efficiency

\footnotetext{
${ }^{4}$ https://www.hpcwire.com/2018/05/07/cavium-announces-ga-for-thunderx2

${ }^{5}$ https://ark.intel.com/content/www/us/en/ark/products/120501/ intel-xeon-platinum-8160-processor-33m-cache-2-10-ghz.html
} 


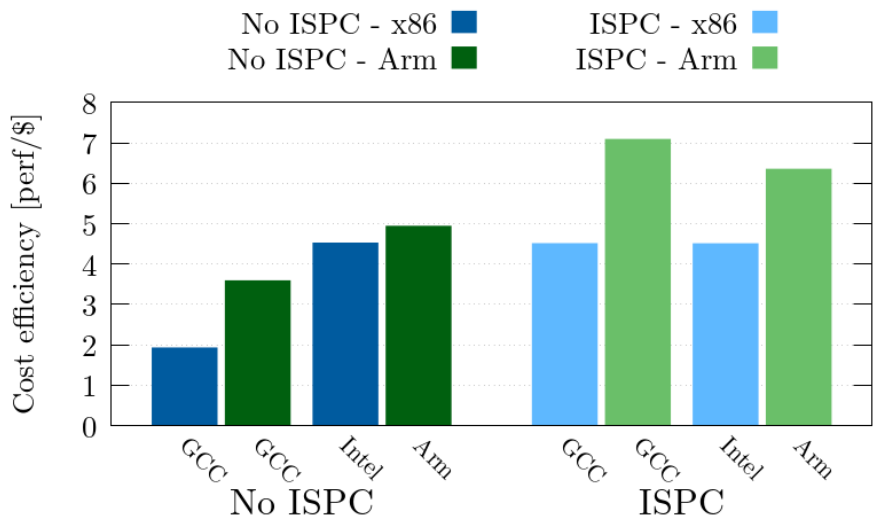

Fig. 10. Cost efficiency of Intel- and Arm-based systems

achieved by the Arm system is between $41 \%$ and $57 \%$ higher than the Intel-based system.

The performance figures presented in Section IV-A do not make the Armv8 Marvell ThunderX2 solution shine compared with state-of-the-art HPC platforms. Its equivalent energy budget and its higher cost efficiency, however, make it attractive for HPC deployment, especially if we put these figures in the perspective of the recent and future technological development by Arm-based CPU manufacturers.

\section{CONCLusions}

In this paper, we presented an evaluation of a neural simulation called CoreNEURON on two HPC platforms powered by two different architectures: Intel and Arm. CoreNEURON is a complex neuroscience simulation code leveraging a DSL (NMODL) and a source-to-source compiler (MOD2C). Those underlying layers have been previously extended to support different architectures, especially SIMD units of x86 and Arm via the ISPC backend.

Our paper shows the benefit of using tools taking care of the data parallelism. In all cases where ISPC has been applied, we observed great performance benefits with speedups ranging from $1.2 \times$ to $2.3 \times$ independently from the architecture. We consider this a great example of decoupling the optimization targeting the architecture from the scientific application. Approaches like the one chosen by CoreNEURON should be promoted, especially nowadays, when vector architectures are becoming more and more critical for the HPC community. The performance gain due to the vectorization is often coupled with the memory management of the system and the memory footprint of the application. We left the analysis of memory usage for future work, where we can include different memory technologies.

More concerning the Arm ecosystem, our comparison shows that: i) The software ecosystem is ready: vendor-specific compilers deliver in general better performance than GCC, and this is true also for the Arm HPC compiler. Also, the whole Arm ecosystem is ready to support the compilation and the execution of a complex software infrastructure like the one required by a neural simulation. ii) The raw performance of the
Armv8 Marvell ThunderX2 CPU is between $1.4 \times$ and $1.8 \times$ slower than the x86 Intel Skylake ones with the CoreNEURON simulation under study. iii) Interestingly enough, the energy consumption is the same for all the best performing cases on both architectures, showing a potential for excellent energy efficiency for future generations of chips. $i v$ ) The strong point of Arm being the cost efficiency: combining, in fact, performance and price shows that the Armv8 Marvell ThunderX2based system is between $1.3 \times$ and $1.5 \times$ more cost-efficient than state-of-the-art $x 86$ ones powered by Intel Skylake.

In this study, we used a synthetic model of ringtest network designed to analyze the CoreNEURON simulator's performance. As future work, we will apply this methodology and extend our analysis for large scale simulation of the hippocampus model that is being built as part of the Human Brain Project.

\section{ACKNOWLEDGMENTS}

This work is partially supported by the Spanish Government (SEV-2015-0493), by the Spanish Ministry of Science and Technology (TIN2015-65316-P), by the Generalitat de Catalunya (2017-SGR-1414), by the European Mont-Blanc 3 project (GA n. 671697), Human Brain Project SGA2 (GA n. 785907), and POP CoE (GA n. 824080).

\section{REFERENCES}

[1] M. L. Hines and N. T. Carnevale, "The NEURON simulation environment." Neural computation, 1997.

[2] H. E. Plesser et al., "Efficient parallel simulation of large-scale neuronal networks on clusters of multiprocessor computers," Euro-Par 2007 Parallel Processing, vol. 4641, pp. 672-681, 2007.

[3] S. Wils and E. De Schutter, "STEPS: Modeling and simulating complex reaction-diffusion systems with Python," Frontiers in Neuroinformatics, vol. 3, p. 15, 2009.

[4] P. Kumbhar et al., "CoreNEURON : An Optimized Compute Engine for the NEURON Simulator," Frontiers in Neuroinformatics, vol. 13, 2019.

[5] — "An optimizing multi-platform source-to-source compiler framework for the neuron modeling language," in International Conference on Computational Science. Springer, 2020, pp. 45-58.

[6] M. Pharr and W. R. Mark, "Ispc: A SPMD compiler for highperformance CPU programming," in 2012 Innovative Parallel Computing (InPar). San Jose, CA, USA: IEEE, May 2012, pp. 1-13.

[7] S. D. Hammond et al., "The Astra Supercomputer," Sandia National Lab, Tech. Rep., 2018. [Online]. Available: https://www.osti.gov/ servlets/purl/1574565

[8] D. DAgostino et al., "SoC-based computing infrastructures for scientific applications and commercial services: Performance and economic evaluations," Future Generation Computer Systems, vol. 96, pp. 11-22, Jul. 2019.

[9] M. Jarus, S. Varrette, A. Oleksiak, and P. Bouvry, "Performance Evaluation and Energy Efficiency of High-Density HPC Platforms Based on Intel, AMD and ARM Processors," in Energy Efficiency in Large Scale Distributed Systems, 2013, pp. 182-200.

[10] E. Calore et al., "ThunderX2 Performance and Energy-Efficiency for HPC Workloads," Computation, vol. 8, no. 1, p. 20, 2020.

[11] M. Garcia-Gasulla, F. Mantovani, M. Josep-Fabrego, B. Eguzkitza, and G. Houzeaux, "Runtime mechanisms to survive new HPC architectures: a use case in human respiratory simulations," Int. J. High Perform. Comput. Appl., vol. 34, no. 1, pp. 42-56, 2020.

[12] S. Sprenger, S. Zeuch, and U. Leser, "Exploiting Automatic Vectorization to Employ SPMD on SIMD Registers," in 2018 IEEE 34th International Conference on Data Engineering Workshops (ICDEW), Apr. 2018, pp. 90-95, iSSN: 2473-3490. 
[13] R. Kramer and G. Rünger, "Performance and efficiency investigations of simd programs of coulomb solvers on multi-and many-core systems with vector units," in 2020 28th Euromicro International Conference on Parallel, Distributed and Network-Based Processing (PDP). IEEE, 2020, pp. 237-244.

[14] C. Gómez Crespo, M. Casas Guix, F. Mantovani, and E. Focht, "Optimizing sparse matrix-vector multiplication in NEC SX-Aurora vector engine," 2020. [Online]. Available: https://upcommons.upc.edu/ handle/2117/192586

[15] N. Stephens et al., "The ARM scalable vector extension," IEEE Micro, vol. 37, no. 2, pp. 26-39, 2017.

[16] M. L. Hines and N. T. Carnevale, "Expanding NEURON's repertoire of mechanisms with NMODL," Neural computation, vol. 12, pp. 995-1007, 2000.

[17] T. Ewart, F. Delalondre, and F. Schürmann, "Cyme: a library maximizing SIMD computation on user-defined containers," in International Supercomputing Conference. Springer, 2014, pp. 440-449.

[18] [Online]. Available: https://github.com/nrnhines/ringtest

[19] F. Mantovani, M. Garcia-Gasulla et al., "Performance and energy consumption of HPC workloads on a cluster based on Arm ThunderX2 CPU," Future Generation Computer Systems, 2020.

[20] F. Banchelli, M. Garcia-Gasulla, G. Houzeaux, and F. Mantovani, "Benchmarking of state-of-the-art HPC Clusters with a Production CFD Code," in Proceedings of the Platform for Advanced Scientific Computing Conference, 2020, pp. 1-11.

[21] G. Llort et al., "On the usefulness of object tracking techniques in performance analysis," in SC'13: Proceedings of the Int. Conf. on High Performance Computing, Networking, Storage and Analysis, 2013.

[22] P. J. Mucci, S. Browne, C. Deane, and G. Ho, "Papi: A portable interface to hardware performance counters," in Proceedings of the department of defense HPCMP users group conference, vol. 710, 1999.

[23] S. McIntosh-Smith et al., "A performance analysis of the first generation of HPC-optimized Arm processors," Concurrency and Computation: Practice and Experience, vol. 31, no. 16, 2019. 